\title{
Association of a butyrophilin, subfamily 2, member A1 gene polymorphism with hypertension
}

\author{
YOSHIKO MURAKATA $^{1,2}$, TETSUO FUJIMAKI ${ }^{3}$ and YOSHIJI YAMADA ${ }^{1,2}$ \\ ${ }^{1}$ Department of Human Functional Genomics, Life Science Research Center; \\ ${ }^{2}$ Department of Medical Genomics and Proteomics, Institute of Basic Sciences, Graduate School of Medicine, Mie University, \\ Tsu, Mie 514-8507; ${ }^{3}$ Department of Cardiovascular Medicine, Inabe General Hospital, Inabe, Mie 511-0428, Japan
}

Received June 24, 2014; Accepted July 25, 2014

DOI: 10.3892/br.2014.340

\begin{abstract}
The $\mathrm{C} \rightarrow \mathrm{T}$ polymorphism (rs6929846) of the butyrophilin, subfamily 2, member A1 (BTN2A1) gene has been previously identified as a susceptibility locus for myocardial infarction by a genome-wide association study. As hypertension is a major risk factor for myocardial infarction, the association between the BTN2Al polymorphism, rs6929846, and myocardial infarction may be partly due to its effect on hypertension susceptibility. The aim of the present study was to examine the possible association of rs6929846 with hypertension. The study subjects comprised 5,959 community-dwelling individuals $(2,183$ subjects with hypertension and 3,776 controls) who were recruited to a population-based cohort study. The rs6929846 genotype was determined by a method that combined polymerase chain reaction and sequence-specific oligonucleotide probes with suspension array technology. Comparisons between the genotype distributions $(\mathrm{P}=0.0090)$ and allele frequencies $(\mathrm{P}=0.0051)$ by the $\chi^{2}$ test revealed that rs6929846 was significantly associated with hypertension. Multivariable logistic regression analysis with adjustment for age, gender, body mass index and smoking status revealed that rs6929846 was significantly associated with hypertension $(\mathrm{P}=0.0008$; odds ratio, 1.29 ; dominant model), with the minor $T$ allele representing a risk factor for this condition. Among all the individuals, systolic, diastolic and mean blood pressure was significantly higher in the combined group of individuals with the $C T$ or $T T$ genotypes compared to the $C C$ genotype group. BTN2Al may thus be a susceptibility gene for hypertension. Therefore, determining the genotype for this polymorphism may provide genetic risk assessment information for hypertension.
\end{abstract}

Correspondence to: Professor Yoshiji Yamada, Department of Human Functional Genomics, Life Science Research Center, Mie University, 1577 Kurima-machiya Road, Tsu, Mie 514-8507, Japan E-mail: yamada@gene.mie-u.ac.jp

Key words: hypertension, genetics, polymorphism, cohort study, genetic epidemiology

\section{Introduction}

Hypertension is a complex multifactorial disorder that is believed to result from an interaction between the genetic background of an individual and various environmental factors (1). As hypertension is a significant risk factor for coronary artery disease, stroke and end-stage renal disease, prevention of hypertension in individuals is a crucial aim. One approach to prevention and selection of the most appropriate hypertension treatment is to identify disease susceptibility genes. Previous genome-wide association studies (GWAS) have implicated various loci and genes in predisposition to hypertension in Caucasian and African-American populations (2-7). An adducin 2 gene polymorphism (rs3755351) was shown to be a susceptibility locus for hypertension in Japanese individuals (8), however, the genes that confer susceptibility to this condition in Japanese individuals remains to be definitively identified.

Our previous GWAS showed that the $\mathrm{C} \rightarrow \mathrm{T}$ polymorphism (rs6929846) in the 5' untranslated region of the butyrophilin, subfamily 2, member A1 (BTN2A1) gene was significantly associated with myocardial infarction in Japanese individuals (9). As hypertension is a major risk factor for the development of atherosclerotic disease, including myocardial infarction, it was hypothesized that the rs6929846 polymorphism of BTN2A1 may contribute to the genetic susceptibility to myocardial infarction through affecting the predisposition to hypertension. The aim of the present study was to examine a possible association of rs6929846 with hypertension in community-dwelling Japanese individuals.

\section{Patients and methods}

Study population. The study subjects comprised 5,959 community-dwelling Japanese individuals $(2,183$ subjects with hypertension, 3,776 controls) who were recruited to a population-based cohort study in Inabe (Mie, Japan) between March 2010 and September 2012 (Inabe Health and Longevity Study). The subjects with hypertension had a systolic blood pressure (BP) and/or diastolic BP of $\geq 140$ or $\geq 90 \mathrm{mmHg}$, respectively, or were not on antihypertensive medication. Individuals with valvular heart disease, congenital malformations of the heart or vessels, or renal or endocrinological diseases that cause secondary hypertension were excluded from the study. The 
Table I. Characteristics of 5,959 study subjects.

\begin{tabular}{|c|c|c|c|}
\hline Characteristic & Hypertension & Control & P-value \\
\hline Subjects, $\mathrm{n}$ & 2183 & 3776 & \\
\hline Age, years & $60.9 \pm 10.6$ & $50.1 \pm 12.4$ & $<0.0001$ \\
\hline Gender, male/female (\%) & $62.3 / 37.7$ & $48.6 / 51.4$ & $<0.0001$ \\
\hline Body mass index, $\mathrm{kg} / \mathrm{m}^{2}$ & $24.1 \pm 3.6$ & $22.3 \pm 3.1$ & $<0.0001$ \\
\hline Current or former smoker, $\%$ & 47.7 & 44.5 & 0.0161 \\
\hline Coronary artery disease, $\%$ & 4.6 & 0.8 & $<0.0001$ \\
\hline Stroke, ischemic and hemorrhagic, $\%$ & 4.1 & 1.1 & $<0.0001$ \\
\hline Systolic blood pressure, $\mathrm{mmHg}$ & $133 \pm 15$ & $113 \pm 11$ & $<0.0001$ \\
\hline Diastolic blood pressure, $\mathrm{mmHg}$ & $83 \pm 12$ & $70 \pm 10$ & $<0.0001$ \\
\hline Mean blood pressure, $\mathrm{mmHg}$ & $99 \pm 12$ & $84 \pm 9$ & $<0.0001$ \\
\hline Pulse pressure, $\mathrm{mmHg}$ & $50 \pm 13$ & $43 \pm 9$ & $<0.0001$ \\
\hline Dyslipidemia, $\%$ & 76.6 & 54.8 & $<0.0001$ \\
\hline Serum total cholesterol, $\mathrm{mmol} / \mathrm{l}$ & $5.23 \pm 0.88$ & $5.24 \pm 0.88$ & 0.5910 \\
\hline Serum triglycerides, mmol/1 & $1.44 \pm 0.96$ & $1.21 \pm 0.78$ & $<0.0001$ \\
\hline Serum HDL-cholesterol, mmol/1 & $1.60 \pm 0.44$ & $1.70 \pm 0.45$ & $<0.0001$ \\
\hline Serum LDL-cholesterol, mmol/1 & $3.17 \pm 0.79$ & $3.19 \pm 0.81$ & 0.2140 \\
\hline Diabetes mellitus, $\%$ & 20.1 & 6.7 & $<0.0001$ \\
\hline Fasting plasma glucose, $\mathrm{mmol} / \mathrm{l}$ & $5.83 \pm 1.26$ & $5.40 \pm 0.96$ & $<0.0001$ \\
\hline Blood hemoglobin $\mathrm{A}_{1 \mathrm{c}}, \%$ & $5.83 \pm 0.73$ & $5.59 \pm 0.59$ & $<0.0001$ \\
\hline Serum creatinine, $\mu \mathrm{mol} / 1$ & $70.5 \pm 20.3$ & $64.7 \pm 13.9$ & $<0.0001$ \\
\hline
\end{tabular}

Quantitative data are mean \pm standard deviation. Dyslipidemia, serum triglyceride concentration $\geq 1.65 \mathrm{mmol} / \mathrm{l}$, serum high-density lipoprotein (HDL)-cholesterol $<1.04 \mathrm{mmol} / 1$, serum low-density lipoprotein (LDL)-cholesterol $\geq 3.64 \mathrm{mmol} / \mathrm{l}$ or undergoing antidyslipidemic medication; diabetes mellitus, fasting plasma glucose concentration $\geq 6.93 \mathrm{mmol} / \mathrm{l}$, hemoglobin $\mathrm{A}_{1 \mathrm{c}}$ content $\geq 6.5 \%$ or undergoing antidiabetes medication.

control individuals had systolic BP and diastolic BP of $<140$ and $<90 \mathrm{mmHg}$, respectively, and no history of hypertension or of antihypertensive medication. BP was measured at least twice with subjects having rested in the sitting position for $>5 \mathrm{~min}$. The measurements were documented by a skilled physician or a nurse according to the guidelines of the American Heart Association (10). The study protocol complied with the Declaration of Helsinki and was approved by the Committees for the Ethics of Human Research of Mie University Graduate School of Medicine and Inabe General Hospital. Written informed consent was obtained from all the subjects.

Polymorphism genotyping. Venous blood $(5 \mathrm{ml})$ was collected into tubes containing $50 \mathrm{mmol} / \mathrm{l}$ ethylenediaminetetraacetic acid (disodium salt), the peripheral blood leukocytes were isolated and genomic DNA was extracted from these cells with a DNA extraction kit (SMITEST EX-R\&D; Medical and Biological Laboratories, Co., Ltd., Nagoya, Japan). Genotypes of rs6929846 were determined at G\&G Science Co., Ltd., (Fukushima, Japan) by a method that combined the polymerase chain reaction and sequence-specific oligonucleotide probes with suspension array technology (Luminex, Austin, TX, USA), as described previously $(11,12)$. Detailed genotyping methodology was also described previously (13).

Statistical analysis. Quantitative data were compared between the subjects with hypertension and the controls by the unpaired Student's t-test. Categorical data were compared by the $\chi^{2}$ test.
The gene counting method estimated the allele frequencies and the $\chi^{2}$ test was used to identify departure from Hardy-Weinberg equilibrium. Multivariable logistic regression analysis was performed with hypertension as a dependent variable and independent variables, including age, gender ( 0 , female; 1 , male), body mass index (BMI), smoking status (0, non-smoker; 1 , current or former smoker) and rs6929846 genotype; and the P-value, odds ratio (OR) and 95\% confidence interval (CI) were calculated. The rs6929846 genotype was assessed according to dominant ( 0 , wild-type homozygotes; 1 , the combined group of variant homozygotes and heterozygotes) and recessive ( 0 , the combined group of wild-type homozygotes and heterozygotes; 1 , variant homozygotes) genetic models. $\mathrm{P}<0.05$ was considered to indicate a statistically significant difference. Statistical tests were performed with the JMP 5.1 software (SAS Institute, Inc., Cary, NC, USA).

\section{Results}

Characteristics. The characteristics of the 5,959 study subjects are shown in Table I. Age, the frequency of males, BMI, the prevalence of smoking, coronary artery disease, stroke, dyslipidemia and diabetes mellitus, as well as serum concentrations of triglycerides and creatinine, fasting plasma glucose level and blood glycosylated hemoglobin (hemoglobin $\mathrm{A}_{1 \mathrm{c}}$ ) content, were increased, whereas serum concentrations of high-density lipoprotein (HDL)-cholesterol were lower, in subjects with hypertension compared to the controls. 
Table II. Comparison of the genotype distributions and allele frequencies of the butyrophilin, subfamily 2, member A1 (BTN2A1) gene polymorphism, rs6929846, by the $\chi^{2}$ test between subjects with hypertension and the controls.

\begin{tabular}{|c|c|c|c|c|c|}
\hline Gene & Polymorphism & Hypertension (\%) & Controls (\%) & P-value (genotype) & P-value (allele) \\
\hline \multirow[t]{4}{*}{$B T N 2 A 1$} & rs6929846 & & & 0.0090 & 0.0051 \\
\hline & $C C$ & $1693(77.6)$ & $3051(80.8)$ & & \\
\hline & $C T$ & $462(21.2)$ & $677(17.9)$ & & \\
\hline & $T T$ & $28(1.3)$ & $47(1.2)$ & & \\
\hline Hardy-Weinberg P-value & & 0.5765 & 0.1754 & & \\
\hline
\end{tabular}

Table III. Multivariable logistic regression analysis of the BTN2A1 polymorphism, rs6929846, and hypertension.

\begin{tabular}{|c|c|c|c|c|c|}
\hline \multirow[b]{2}{*}{ Gene } & \multirow[b]{2}{*}{ Polymorphism $(\mathrm{C} \rightarrow \mathrm{T})$} & \multicolumn{2}{|c|}{ Dominant } & \multicolumn{2}{|c|}{ Recessive } \\
\hline & & P-value & OR $(95 \% \mathrm{CI})$ & P-value & OR $(95 \% \mathrm{CI})$ \\
\hline$B T N 2 A 1$ & rs6929846 & 0.0008 & $1.29(1.11-1.50)$ & 0.8582 & $1.05(0.60-1.79)$ \\
\hline
\end{tabular}

Multivariable logistic regression analysis was performed with adjustment for age, gender, body mass index and smoking status. BTN2A1, butyrophilin, subfamily 2, member A1 gene. OR, odds ratio; CI, confidence interval.

Table IV. Association of the BTN2A1 polymorphism, $r s 6929846$, with systolic, diastolic or mean blood pressure, or pulse pressure.

\begin{tabular}{|c|c|c|c|c|c|}
\hline \multirow[b]{2}{*}{ rs6929846 individuals } & \multicolumn{3}{|c|}{ Genotype } & \multirow{2}{*}{$\begin{array}{c}\mathrm{P} \text {-value } \\
(C C \text { vs. } C T+T T)\end{array}$} & \multirow{2}{*}{$\begin{array}{c}\text { P-value } \\
(C C+C T \text { vs. } T T)\end{array}$} \\
\hline & $C C$ & $C T$ & $T T$ & & \\
\hline \multicolumn{6}{|l|}{ All } \\
\hline Subjects, $\mathrm{n}$ & 4721 & 1134 & 75 & & \\
\hline Systolic blood pressure, $\mathrm{mmHg}$ & $120 \pm 16$ & $121 \pm 16$ & $123 \pm 16$ & $0.0390^{\mathrm{a}}$ & 0.1100 \\
\hline Diastolic blood pressure, $\mathrm{mmHg}$ & $74 \pm 12$ & $75 \pm 12$ & $76 \pm 12$ & $0.0045^{\mathrm{a}}$ & 0.4342 \\
\hline Mean blood pressure, $\mathrm{mmHg}$ & $90 \pm 12$ & $91 \pm 13$ & $92 \pm 12$ & $0.0069^{\mathrm{a}}$ & 0.2389 \\
\hline Pulse pressure, $\mathrm{mmHg}$ & $46 \pm 11$ & $46 \pm 11$ & $48 \pm 11$ & 0.8956 & 0.1430 \\
\hline \multicolumn{6}{|l|}{ Not on antihypertensive medication } \\
\hline Subjects, $\mathrm{n}$ & 3709 & 850 & 59 & & \\
\hline Systolic blood pressure, $\mathrm{mmHg}$ & $117 \pm 15$ & $118 \pm 16$ & $120 \pm 16$ & 0.0657 & 0.1577 \\
\hline Diastolic blood pressure, $\mathrm{mmHg}$ & $73 \pm 12$ & $74 \pm 12$ & $75 \pm 11$ & $0.0067^{\mathrm{a}}$ & 0.3204 \\
\hline Mean blood pressure, $\mathrm{mmHg}$ & $88 \pm 12$ & $89 \pm 13$ & $90 \pm 12$ & $0.0116^{\mathrm{a}}$ & 0.2220 \\
\hline Pulse pressure, $\mathrm{mmHg}$ & $45 \pm 10$ & $44 \pm 10$ & $46 \pm 10$ & 0.6717 & 0.3445 \\
\hline
\end{tabular}

${ }^{\mathrm{a}} \mathrm{P}<0.05$. Data for blood pressure and pulse pressure are mean \pm standard deviation. BTN2A1, butyrophilin, subfamily 2, member A1 gene.

rs6929846 associations. Comparisons of the genotype distributions and allele frequencies by the $\chi^{2}$ test between the subjects with hypertension and the controls revealed that rs6929846 of the BTN2Al gene was significantly $(\mathrm{P}<0.05)$ associated with hypertension (Table II). The genotype distributions of rs6929846 were in Hardy-Weinberg equilibrium among the subjects with hypertension and the controls (Table II).

Multivariable logistic regression analysis with adjustment for age, gender, BMI and smoking status revealed that rs6929846 (dominant model) was significantly associated with hypertension, with the minor $T$ allele representing a risk factor (Table III).
The association of rs6929846 with systolic, diastolic or mean BP, or pulse pressure was also examined among all the individuals or individuals not on antihypertensive medication. Among all the individuals, systolic, diastolic and mean BP was significantly higher in the combined group of individuals with the $C T$ or $T T$ genotype compared to those with the $C C$ genotype. Among the individuals not on antihypertensive medication, diastolic and mean, but not systolic BP, were significantly higher in the combined group of individuals with the $C T$ or $T T$ compared to those with the $C C$ genotype. There was no significant difference in pulse pressure between the rs6929846 genotypes (Table IV). 


\section{Discussion}

The present study has shown that the rs6929846 polymorphism of the BTN2Al gene was significantly associated with the prevalence of hypertension in community-dwelling Japanese individuals, with the minor $T$ allele representing a risk factor for this condition. Our previous study showed that rs6929846 was significantly associated with hypertension in a different hospital-based study population (14). The results of the present population-based study are consistent with the previous observations in the hospital-based study (14) and validated the association of rs6929846 in BTN2Al with hypertension.

BTN2A1 is a cell surface transmembrane glycoprotein that is a member of the butyrophilin superfamily. The butyrophilin family was originally identified due to its ability to induce the production of milk fat globules (15), however, a number of proteins belonging to the butyrophilin and butyrophilin-like families were shown to regulate immune function and polymorphisms in the protein coding sequences were associated with the predisposition to inflammatory diseases (16). Our previous study showed that the $T$ allele of the rs 6929846 polymorphism of the $B T N 2 A 1$ gene was associated with an increased risk of myocardial infarction and with an increased transcription activity of BTN2A1 (9). The serum high sensitivity C-reactive protein concentrations were significantly increased in the individuals in the combined group of $C T$ or $T T$ compared to those with the $C C$ genotype among healthy individuals without neoplastic, infectious or inflammatory disease $(9,17)$. Therefore, the $T$ allele of rs6929846 may accelerate the inflammatory processes.

Previous studies have indicated that chronic vascular inflammation influences BP and vascular remodeling (18-21). Systolic and diastolic BP and pulse pressure have been shown to be positively associated with interleukin-6 plasma concentrations in healthy males (18). Plasma concentrations of high sensitivity C-reactive protein were greater in individuals with hypertension compared to those with normal BP, and were positively correlated with systolic BP and pulse pressure (19). Oxidative stress and vascular inflammation have been shown to affect BP, and chronic inflammation may play a critical role in the pathogenesis of hypertension $(20,21)$. The present study showed that rs6929846 of BTN2Al was significantly associated with hypertension, with the minor $T$ allele representing a risk factor for this condition. The enhancement of chronic inflammation by the $T$ allele of rs6929846 may account for its association with hypertension, although the molecular mechanism of the affect of rs6929846 on the development of hypertension remains to be elucidated.

In the results of the present study, systolic, diastolic or mean BP were increased by $2-3 \mathrm{mmHg}$ in individuals with the $T T$ genotype compared to those with the $C C$ genotype. Such a difference is small at the individual level and may not have practical clinical implications. However, this increase in BP is important at the population level, due to the high incidence of coronary artery disease and stroke. The reduction in mortality estimated for each 2-mmHg decrease in systolic BP is $4 \%$ for coronary artery disease and $6 \%$ for stroke (22). Small differences in the average BP indicate significant differences in population mortality (22). In Japan, the reduction of morbidity or mortality estimated for each $2-\mathrm{mmHg}$ decrease in systolic BP for coronary artery disease is $5.4 \%$ (5,367 individuals) or $4.0 \%$ (3,944 individuals), respectively, and for stroke it is $6.4 \%$ (19,757 individuals) or 3.0\% (9,127 individuals), respectively (23). Therefore, identification of genetic variants that contribute to the increased risk of hypertension is clinically important.

There were limitations in the present study: i) As the study subjects comprised of only Japanese individuals, further study is required in other ethnic groups. ii) rs6929846 of BTN2Al is possibly in linkage disequilibrium with other polymorphisms in $B T N 2 A 1$ or in other nearby genes, which are responsible for hypertension development. iii) The functional relevance of rs6929846 of BTN2Al to pathogenesis of hypertension remains unclear.

In conclusion, the results of the present study indicate that BTN2A1 may be a susceptibility gene for hypertension in Japanese individuals. Determining the rs6929846 genotype may provide genetic risk assessment informative for hypertension. As multiple variants, which each have a small effect, are believed to be responsible for a large fraction of the genetic component of essential hypertension, further identification of hypertension susceptibility genes will allow more accurate assessment of the genetic component of this condition.

\section{Acknowledgements}

The present study was supported by research grants from the Japan Health Foundation and Okasan Kato Culture Promotion Foundation (to Y.Y.).

\section{References}

1. Lifton RP, Gharavi AG and Geller DS: Molecular mechanisms of human hypertension. Cell 104: 545-556, 2001.

2. Wellcome Trust Case Control Consortium: Genome-wide association study of 14,000 cases of seven common diseases and 3,000 shared controls. Nature 447: 661-678, 2007.

3. Newton-Cheh C, Johnson T, Gateva V, et al: Genome-wide association study identifies eight loci associated with blood pressure. Nat Genet 41: 666-676, 2009.

4. Levy D, Ehret GB, Rice K, et al: Genome-wide association study of blood pressure and hypertension. Nat Genet 41: 677-687, 2009.

5. Org E, Eyheramendy S, Juhanson P, et al: Genome-wide scan identifies $\mathrm{CDH} 13$ as a novel susceptibility locus contributing to blood pressure determination in two European populations. Hum Mol Genet 18: 2288-2296, 2009.

6. Adeyemo A, Gerry N, Chen G, et al: A genome-wide association study of hypertension and blood pressure in African Americans. PLoS Genet 5: e1000564, 2009.

7. Tragante V, Barnes MR, Ganesh SK, et al: Gene-centric meta-analysis in 87,736 individuals of European ancestry identifies multiple blood-pressure-related loci. Am J Hum Genet 94: 349-360, 2014.

8. Kato N, Miyata T, Tabara Y, et al: High-density association study and nomination of susceptibility genes for hypertension in the Japanese National Project. Hum Mol Genet 17: 617-627, 2008.

9. Yamada Y, Nishida T, Ichihara S, et al: Association of a polymorphism of BTN2A1 with myocardial infarction in East Asian populations. Atherosclerosis 215: 145-152, 2011.

10. Perloff D, Grim C, Flack J, Frohlich ED, Hill M, McDonald M and Morgenstern BZ: Human blood pressure determination by sphygmomanometry. Circulation 88: 2460-2470, 1993.

11. Yamada Y, Fuku N, Tanaka M, et al: Identification of CELSR1 as a susceptibility gene for ischemic stroke in Japanese individuals by a genome-wide association study. Atherosclerosis 207: 144-149, 2009.

12. Yamada Y, Nishida T, Ichihara S, et al: Identification of chromosome $3 \mathrm{q} 28$ and ALPK1 as susceptibility loci for chronic kidney disease in Japanese individuals by a genome-wide association study. J Med Genet 50: 410-418, 2013. 
13. Itoh Y, Mizuki N, Shimada T, et al: High-throughput DNA typing of HLA-A, -B, -C, and -DRB1 loci by a PCR-SSOP-Luminex method in the Japanese population. Immunogenetics 57: 717-729, 2005.

14. Horibe H, Kato K, Oguri M, et al: Association of a polymorphism of BTN2A1 with hypertension in Japanese individuals. Am J Hypertens 24: 924-929, 2011.

15. Ogg SL, Weldon AK, Dobbie L, Smith AJ and Mather IH: Expression of butyrophilin (Btn1a1) in lactating mammary gland is essential for the regulated secretion of milk-lipid droplets. Proc Natl Acad Sci USA 101: 10084-10089, 2004.

16. Arnett HA, Escobar SS and Viney JL: Regulation of costimulation in the era of butyrophilins. Cytokine 46: 370-375, 2009.

17. Oguri M, Kato K, Yoshida T, et al: Association of a genetic variant of BTN2A1 with metabolic syndrome in East Asian populations. J Med Genet 48: 787-792, 2011.

18. Chae CU, Lee RT, Rifai N and Ridker PM: Blood pressure and inflammation in apparently healthy men. Hypertension 38 : 399-403, 2001
19. Schillaci G, Pirro M, Gemelli F, et al: Increased C-reactive protein concentrations in never-treated hypertension: the role of systolic and pulse pressures. J Hypertens 21: 1841-1846, 2003.

20. Savoia $C$ and Schiffrin EL: Inflammation in hypertension. Curr Opin Nephrol Hypertens 15: 152-158, 2006.

21. Androulakis ES, Tousoulis D, Papageorgiou N, Tsioufis C, Kallikazaros I and Stefanadis C: Essential hypertension: is there a role for inflammatory mechanisms? Cardiol Rev 17: 216-221, 2009.

22. Stamler J, Rose G, Stamler R, Elliott P, Dyer A and Marmot M: INTERSALT study findings. Public health and medical care implications. Hypertension 14: 570-577, 1989.

23. Ministry of Health, Labour and Welfare of Japan: Kenkounippon 21 (in Japanese). http://www.kenkounippon21.gr.jp/. Accessed June 20, 2014. 